\title{
Photo-activated $\mathrm{NO}_{2}$ Sensors with 1-D nanomaterials Integrated with Micro-LED for High Irradiation Efficiency
}

\author{
Incheol Cho, Minkyu Cho, Kyungnam Kang, and Inkyu Park \\ Department of Mechanical Engineering, KAIST, Daejeon 305-701, S.Korea \\ inkyu@kaist.ac.kr
}

\begin{abstract}
In this paper, we propose a photo-activated metal oxide gas sensor integrated with a commercial $\mu$ LED $\left(\lambda_{\text {peak }}=390 \mathrm{~nm}\right)$ for high photo-irradiation efficiency. The sensing materials, ZnO nanowires or $\mathrm{SnO}_{2}$ nanotubes, were grown on parallel electrodes of Indium Tin Oxide (ITO) patterned on a quartz substrate. The transparency of the ITO electrodes and the quartz substrate allowed the UV LED to be placed beneath the quartz substrate to irradiate the grown nanomaterial, thereby resulting in enhanced gas sensing performance. The $\mathrm{ZnO}$ nanowires or $\mathrm{SnO}_{2}$ nanotubes were directly synthesized by a liquid-phase process on the patterned ITO electrodes for precise positioning and facile assembly of the one-dimensional nanomaterials. Due to the small distance between the photoemitter and the photoreceptor $\left(\mathrm{ZnO}\right.$ or $\left.\mathrm{SnO}_{2}\right)$, the irradiation efficiency is improved in relation to sensors that use external light sources like UV lamps. Furthermore, we have demonstrated that the sensing performance for $\mathrm{NO}_{2}$ gas improves as the intensity of UV light increases.
\end{abstract}

Key words: $\mathrm{ZnO}$ nanowires, $\mathrm{SnO}_{2}$ nanotubes, micro-LED, photo-activated gas sensors, $\mathrm{NO}_{2}$ sensor

\section{Introduction}

Metal oxide gas sensors have been extensively studied for mobile applications due to their small size, high sensitivity, and low cost. Metal oxide gas sensors are generally heated to high temperature $\left(200-400{ }^{\circ} \mathrm{C}\right)$. These high temperatures may cause drift in the sensor signal or lower the long-term reliability due to the thermal degradation of the sensing materials. In contrast, photo-activated gas sensors through UV light irradiation can detect the toxic gases at room temperature. UV irradiation can increase the sensitivity by increasing the number of electron-hole pairs on the surface of the metal oxide, which causes more adsorption of gas molecules, and by promoting the redox reactions of gas molecules on the surface [1]. However, because conventional photocatalytic gas sensors have used external UV lamps or UV LEDs, the irradiation efficiency has been very low due to the long distance between the photo-emitter and the photo-receptor; this, in turn, causes the light sources of these sensors to consume high electrical power.

In this paper, we propose a photo-activated metal oxide gas sensor fully packaged with a commercial $\mu$-LED for high irradiation efficiency. Metal oxide nanomaterials $\left(\mathrm{ZnO}\right.$ or $\left.\mathrm{SnO}_{2}\right)$ were directly synthesized on top of transparent ITO electrodes on a quartz substrate. Different devices based on $\mathrm{ZnO}$ and $\mathrm{SnO}_{2}$, individually, were constructed based on their well-known sensing performance. The metal oxide sensing material is irradiated from the bottom of the transparent quartz substrate by the incorporated commercial $\mu$-LED.

\section{Experimental Method}

The schematic of the complete sensor system is shown in Fig. 1(a). Parallel ITO electrodes were patterned on a quartz substrate through a lift-off process using UV lithography and RF sputtering. The direct synthesis methods for the $\mathrm{ZnO}$ nanowires and the $\mathrm{SnO}_{2}$ nanotubes have been reported in our previous work [2]. The prefabricated ITO electrodes were immersed into a $\mathrm{ZnO}$ precursor (25 $\mathrm{mM}$ zinc nitrate hexahydrate, $25 \mathrm{mM}$ hexamethylenetetramine (HMTA), and 6 $\mathrm{mM}$ polyethyleneimine (PEI) in $\mathrm{DI}$ water). The sensing nanowires are grown on each of the two parallel electrodes by applying a 4.7 voltage (current $=4 \mathrm{~mA}$ ) across the length of each electrode inside the precursor solution; once the nanowires are grown, they interconnect to form an electrical path between these parallel electrodes. This voltage heats up each electrode to around $95^{\circ} \mathrm{C}$ and the $\mathrm{ZnO}$ nanowires are grown along the length of the electrode by a localized hydrothermal reaction. To construct the $\mathrm{SnO}_{2}$-based sensors, $\mathrm{ZnO}$ nanowires were sequentially immersed in $\mathrm{SnO}_{2}$ LPD solution (3.75 mM SnF $2,15 \mathrm{mM} \mathrm{HF}, 7.5$ $\mathrm{mM} \mathrm{H}_{2} \mathrm{O}_{2}$, and $37.5 \mathrm{mM} \mathrm{H}_{3} \mathrm{BO}_{3}$ in $\mathrm{Dl}$ water). $\mathrm{SnO}_{2}$ nanotubes were formed due to the coating of $\mathrm{SnO}_{2}$ and the simultaneous dissolution of $\mathrm{ZnO}$. 
The commercial UV micro-LED (SM0603UV390, BIVAR@, $\lambda_{\text {peak }}=390 \mathrm{~nm}$ ) was placed beneath the above mentioned quartz substrate as shown in Figure 1(a); here, a Silicon base supports the glass substrate through PDMS sidewalls and holds the UV LED in between the sidewalls. We performed gas sensing tests with $\mathrm{NO}_{2}$ gas (1-16 ppm) under UV irradiation with various forward- bias voltages $(2.9,3.2$, and 4.2 $\mathrm{V})$. The electrical resistance of the sensing material was measured with a sourcemeter (Keithley 2636B).

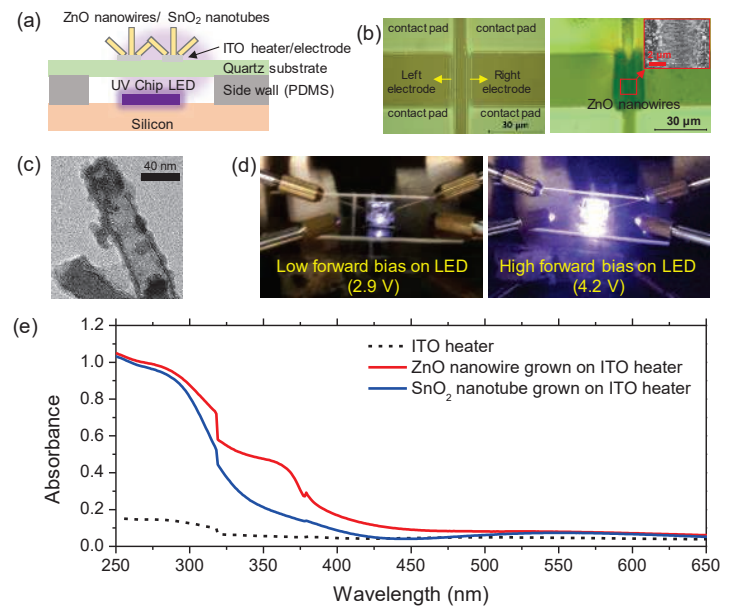

Fig. 1. (a) Cross-sectional view of the sensor device. (b) Photo of the fabricated ITO electrodes and the synthesized $\mathrm{ZnO}$ nanowires. The inset is a SEM image of intercepting nanowires. (c) A TEM image of a $\mathrm{SnO}_{2}$ nanotube. (d) Photo of UV emission in different forward-bias values. (e) Photo-absorption spectrum of $\mathrm{ZnO}$ nanowires and $\mathrm{SnO}_{2}$ nanotubes on the transparent substrate.

\section{Results and Discussion}

Fig.1 (b) shows the fabricated sensor. $\mathrm{ZnO}$ nanowires made junctions and electrical paths between the two parallel electrodes. Fig.1 (c) is a TEM image of a synthesized $\mathrm{SnO}_{2}$ nanotube. Granular and hollow tube structures were well formed. After mounting the quarts substrate with the constructed devices onto the microLED, we visually checked that the luminance of the LED increased with increasing forward bias. Referring to the specifications of the LED, its luminance is 15,100 , and $250 \mathrm{~cd} / \mathrm{m}^{2}$ at $2.9,3.2$, and $4.2 \mathrm{~V}$, respectively. Fig. 1(e) shows the UVVisible light absorption spectrum of the bare ITO electrodes, the electrodes with the grown $\mathrm{ZnO}$ nanowires, and the electrodes with the grown $\mathrm{SnO}_{2}$ nanotubes. Due to the difference of bandgap energy $\left(E_{g, I T O} \sim 4 \mathrm{eV}, \mathrm{E}_{\mathrm{g}, \mathrm{ZnO}} \sim 3.3 \mathrm{eV}\right.$, and $\left.\mathrm{E}_{\mathrm{g}, \mathrm{SnO} 2} \sim 3.6 \mathrm{eV}\right)$, the absorbance of the electrodes with the $\mathrm{ZnO}$ nanowires at $390 \mathrm{~nm}$ wavelength was higher than that of the electrodes with $\mathrm{SnO}_{2}$ nanotubes. Fig.2 shows the results of the gas test. The base resistance of each material decreased as the luminance of the LED increased due to the generated photocurrent. The response speed of the sensors of each material was improved by increasing luminance values of LED. We summarized the sensor response $\left(R^{\prime} / R_{0}\right)$ and the recovery rate (resistance decay ratio at $2000 \mathrm{sec}$ after exposure) in Fig. 3. The sensors based on $\mathrm{SnO}_{2}$ nanotubes showed 4 to 8 times higher response values compared to those of $\mathrm{ZnO}$ nanowires based sensors. The recovery rate of $\mathrm{SnO}_{2}$ nanotube under $\mathrm{V}_{\text {LED }}=4.2 \mathrm{~V}\left(250 \mathrm{~cd} / \mathrm{m}^{2}\right)$ was over $90 \%$.

\section{Conclusion}

We have developed a photo-activated gas sensor using 1D nanomaterials integrated with a micro-LED. Due to the photocatalytic effect of the UV light, the sensing performance (sensitivity, response speed, and recovery rate) at the room temperature was improved, especially in $\mathrm{SnO}_{2}$ nanotube-based sensors.
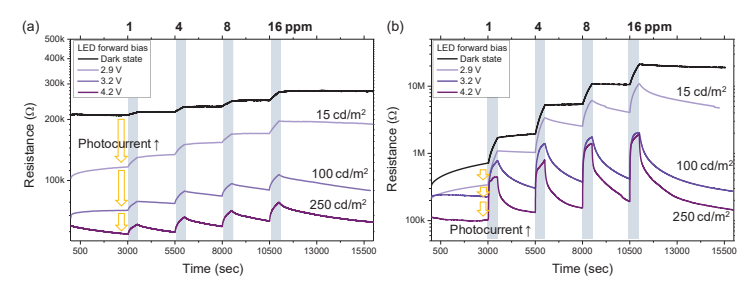

Fig. 2. Dynamic response of (a) ZnO nanowires and (b) $\mathrm{SnO}_{2}$ nanotubes to $\mathrm{NO}_{2}$ gas with UV irradiation.
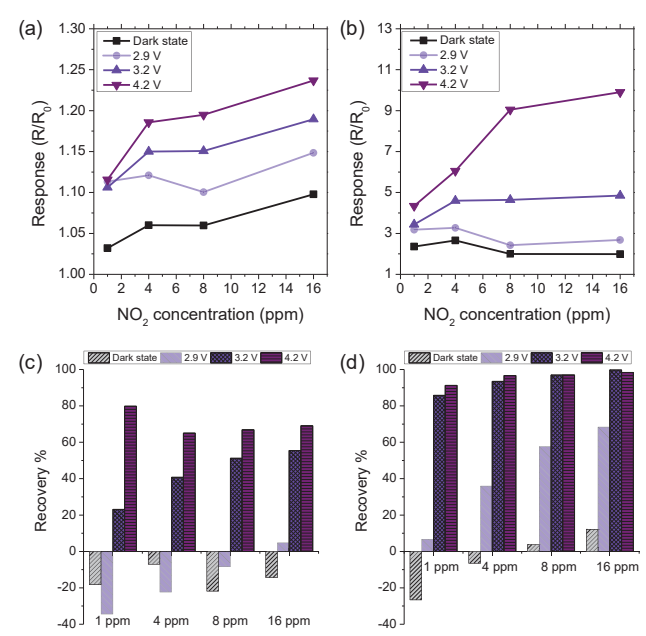

Fig. 3. Summary of sensor responses $\left(R / R_{0}\right)$ under various intensity of irradiation for (a) $\mathrm{ZnO}$ nanowires and (b) $\mathrm{SnO}_{2}$ nanotubes. Recovery ratio at $2000 \mathrm{~s}$ after $\mathrm{NO}_{2}$ exposure of (c) $\mathrm{ZnO}$ nanowires and (d) $\mathrm{SnO}_{2}$ nanotubes.

\section{References}

[1] E. Espid, F. Taghipour, UV-LED Photo-activated Chemical Gas Sensors: A Review, Critical Reviews in Solid State and Materials Sciences 42, 416-432 (2017); doi: 10408436.2016.1226161

[2] I. Cho, K. Kang, D. Yang, J. Yun, I. Park, Localized Liquid-Phase Synthesis of Porous $\mathrm{SnO}_{2}$ Nanotubes on MEMS Platform for LowPower, High Performance Gas Sensors, ACS Applied Materials \& Interfaces 9, 27111-27119 (2017); doi: 10.1021/acsami.7b04850 